Meta

Journal des traducteurs

Translators' Journal

\title{
Conséquences didactiques et théoriques du caractère conventionnel et arbitraire de la traduction des unités phraséologiques
}

\section{Éric Poirier}

Volume 48, numéro 3, septembre 2003

Traduction et enseignement

Translation and teaching

URI : https://id.erudit.org/iderudit/007600ar

DOI : https://doi.org/10.7202/007600ar

Aller au sommaire du numéro

Éditeur(s)

Les Presses de l'Université de Montréal

ISSN

0026-0452 (imprimé)

1492-1421 (numérique)

Découvrir la revue

Citer cet article

Poirier, É. (2003). Conséquences didactiques et théoriques du caractère conventionnel et arbitraire de la traduction des unités phraséologiques. Meta, 48(3), 402-410. https://doi.org/10.7202/007600ar
Résumé de l'article

La traduction des unités phraséologiques (UP) est arbitraire parce que l'équivalence sémantique représente toujours une solution acceptable. Elle est aussi conventionnelle aux deux sens sémiotiques de l'adjectif, c'est-à-dire par relation de référence ou de correspondance, et par calcul sémiotique du sens ou équivalence. Ces caractéristiques de la traduction des UP exigent des méthodes d'enseignement favorisant l'analyse du sens des UP qui s'appuie sur leur délimitation dans la phrase et sur celle de leurs éléments constitutifs. La démonstration du caractère conventionnel de la traduction des UP montre que même si la correspondance et l'équivalence s'opposent en théorie, ces deux procédés peuvent servir en pratique à la traduction d'un même segment linguistique. 


\title{
Conséquences didactiques et théoriques du caractère conventionnel et arbitraire de la traduction des unités phraséologiques
}

\author{
ÉRIC POIRIER \\ Université de Montréal, Montréal, Canada \\ eric.poirier@sympatico.ca
}

\begin{abstract}
RÉSUMÉ
La traduction des unités phraséologiques (UP) est arbitraire parce que l'équivalence sémantique représente toujours une solution acceptable. Elle est aussi conventionnelle aux deux sens sémiotiques de l'adjectif, c'est-à-dire par relation de référence ou de correspondance, et par calcul sémiotique du sens ou équivalence. Ces caractéristiques de la traduction des UP exigent des méthodes d'enseignement favorisant l'analyse du sens des UP qui s'appuie sur leur délimitation dans la phrase et sur celle de leurs éléments constitutifs. La démonstration du caractère conventionnel de la traduction des UP montre que même si la correspondance et l'équivalence s'opposent en théorie, ces deux procédés peuvent servir en pratique à la traduction d'un même segment linguistique.
\end{abstract}

\section{ABSTRACT}

The translation of phraseological units (PUs) is arbitrary because semantic equivalence always represents an acceptable solution. It is also conventional in the two semiotic senses of the word: by the relation of reference or correspondence, and by the semiotic computation of the meaning or equivalence. These characteristics of the translation of PUs require teaching methods focusing on the meaning analysis of PUs that draw on the delimitation of PUs and their constitutive elements. A discussion of the conventional nature of the translation of PUs shows that even though the correspondence and equivalence are theoretical opposites, both processes may be used to translate the same linguistic segment.

\section{MOTS-CLÉS/KEYWORDS}

unité phraséologique, traduction arbitraire, traduction conventionnelle, correspondance, équivalence

Le présent article porte sur les particularités de la traduction des unités phraséologiques (UP) et sur les effets que celles-ci peuvent avoir sur l'enseignement et la théorie de la traduction. Ce travail s'inscrit dans le cadre de la méthodologie d'apprentissage raisonnée élaborée par Delisle (1993), qui a servi de cadre à un cours d'initiation à la traduction. L'expérience acquise au cours de la publication d'un dictionnaire bilingue d'UP (Poirier: 1995) a également servi de toile de fond à cette étude. Nous souhaitons montrer que l'enseignement de la traduction des UP partage un certain nombre de méthodes et d'objectifs avec l'enseignement des langues. Tandis que le caractère conventionnel de la traduction des UP justifie la nécessité de son enseignement et de son apprentissage, la nature arbitraire de la traduction des UP impose en revanche la prise en compte et le respect des conventions d'usage, objectifs que partagent l'enseignement de la traduction et l'enseignement des langues. Bien que les UP

Meta, XLVIII, 3, 2003 
soient elles-mêmes des unités linguistiques arbitraires et conventionnelles parce qu'elles sont des unités lexicales, ce ne sont toutefois pas ces caractéristiques, mais plutôt celles de leur traduction qui sont abordées ici.

Nous définissons les UP comme des unités complexes composées de plusieurs unités lexicales qui ont la particularité d'être lexicalisées. Du fait qu'elles sont complexes, les UP se présentent sous la forme de syntagmes (morphologiques ou syntaxiques) et de phrases lexicalisés. Leur caractère lexicalisé provient du fait qu'elles ont un sens propre. En raison de leur sens propre et de leur nature complexe, les UP sont aussi associées de près à la métaphore.

\section{Comment la traduction peut-elle être arbitraire?}

L'aspect arbitraire de la traduction s'explique par le fait que, pour une même unité linguistique ou un même segment de la langue de départ (LD), il existe toujours plusieurs unités linguistiques ou segments en langue d'arrivée (LA) pour traduire cette unité ou segment. Pour que la relation entre les différentes traductions possibles soit arbitraire, celles-ci doivent avoir sensiblement le même sens, et si ce n'est pas le cas, leurs différences de signification doivent à tout le moins être neutralisées. Pour les unités lexicales, on peut penser par exemple à la traduction de purchase dans la communication commerciale qui se traduit en pratique par "achat», "acquisition » et «approvisionnement», même si «achat» est la traduction la plus généralement acceptée. L'arbitraire traductologique s'inspire en cela de l'arbitraire du signe linguistique selon lequel, comme l'a démontré Benveniste (1966:52), un même objet du monde réel, ou un même référent devrait-on préciser, est en relation sémiotique exclusive avec chacun des différents signes linguistiques qui appartiennent à des langues différentes. Comme l'arbitraire linguistique découle de la prise en compte d'autres langues, l'arbitraire traductologique découle de la prise en compte d'autres traductions. La relation qui se situe au cœur de l'arbitraire traductologique est celle qui existe entre différents segments de la LA les uns par rapport aux autres, et non pas, par exemple, entre le référent de la LD et le référent ou le signe de la LA, ni entre chacun des segments en LA et le segment en LD. Dans ce dernier cas, la relation qui existe entre chacun des segments en LA et le segment en LD n'est pas arbitraire mais conventionnelle, comme nous le verrons ci-après.

\section{Comment la traduction peut-elle être conventionnelle?}

La nature conventionnelle de la traduction provient notamment du fait que le travail des traducteurs est un acte de communication régi dans la société par des règles et des rapports socioéconomiques. Il ne fait aucun doute que dans toute l'histoire de la traduction, jamais le travail des traducteurs n'aura autant été évalué: par l'employé salarié, réviseur ou traducteur, ou la direction de l'entreprise, qui traite avec les pigistes, par les collègues réviseurs des traducteurs salariés, par les clients eux-mêmes et par les utilisateurs de la traduction, pour ne nommer que ces principaux intervenants. De sorte que la traduction comme résultat peut être en elle-même conventionnelle, c'est-à-dire qu'elle correspond à une traduction ayant déjà été reconnue et acceptée par l'organisme ou l'entreprise en question, ou alors par le client ou les sources du traducteur, et c'est cet aspect de la traduction qu'exploitent les mémoires de traduction 
et les dictionnaires bilingues. Lorsque la traduction n'est pas conventionnelle dans ce sens où elle existe déjà, soit parce qu'elle revêt une originalité expressive ou parce qu'elle traduit des textes inédits, elle demeure néanmoins conventionnelle par les procédés de traduction qui ont permis de la créer. Bien plus, dans le cadre des textes pragmatiques, les procédés de traduction utilisés pour traduire des expressions ou des segments dont la traduction n'existe pas doivent être non seulement conventionnels, mais également transparents pour faciliter leur appréciation par tous les intervenants qui doivent justement évaluer la qualité de la traduction.

Il n'y a pas de consensus quant à la nature et au nombre de procédés de traduction. Même s'il s'intéresse surtout aux processus cognitifs de traduction et non pas spécifiquement aux procédés de traduction, Delisle (1993) identifie deux principaux procédés de traduction: la correspondance et l'équivalence ${ }^{1}$. Il est possible d'associer les correspondances au premier type de traduction conventionnelle défini ci-dessus, c'est-à-dire à celle qui est reconnue et acceptée comme telle et qui fait l'objet de la description lexicographique bilingue. La définition de l'équivalence de Jean Delisle diverge toutefois d'une conception suivant laquelle l'équivalence est un procédé conventionnel de traduction. En effet, le statut de l'équivalence revêt chez l'auteur une signification particulière qui stipule qu'elle est toujours étroitement dépendante d'un contexte discursif particulier, l'auteur prenant même soin de signaler dans son glossaire que l'expression «équivalence discursive» est tautologique. Toutefois, la traduction des UP remet en question cette restriction sur l'équivalence du simple fait que les UP sont des unités lexicales et qu'elles se traduisent le plus souvent par équivalence, comme l'ont signalé à juste titre Vinay et Darbelnet (1958: 52). Ces auteurs ont plutôt proposé une typologie regroupant sept procédés de traduction différents répartis en deux principaux procédés de traduction directs et obliques. Cette autre typologie, et l'opposition entre la correspondance et l'équivalence qui en découle, repose en revanche sur le critère de la littéralité dans la définition des procédés de traduction, alors que ce critère relève plutôt d'une comparaison a posteriori de segments linguistiques, et ne permet pas de décrire en tant que telle l'activité de transfert proprement dite.

Plutôt que d'aborder le problème de la définition de l'équivalence sous l'angle du rapport au contexte ou à la littéralité, on peut envisager de redéfinir l'équivalence comme un procédé de traduction conventionnel qui expliquerait mieux les particularités de la traduction des UP et qui rendrait compte du même coup de la similitude entre l'enseignement de la traduction des UP et l'enseignement des langues. Bien que l'apprentissage de la traduction et l'apprentissage d'une langue étrangère présentent des objectifs tout à fait différents et que l'apprentissage de la traduction implique la transmission de connaissances qui lui sont propres, l'enseignement de la traduction des UP pourrait s'inspirer des méthodes pédagogiques de l'enseignement des langues pour améliorer et perfectionner les programmes de formation en traduction, surtout dans l'adéquation entre la théorie et la pratique de la traduction.

D'un point de vue sémiotique et théorique, les deux aspects conventionnels de la traduction évoqués précédemment, c'est-à-dire la traduction conventionnelle comme résultat et en vertu du procédé qui a permis de l'établir, peuvent être rattachés à la distinction entre deux sens de l'adjectif conventionnel établie dans l'Encyclopedic Dictionary of Semiotics (1986) publié sous la direction de Thomas A. Sebeok, à l'article «Sign». On y définit ainsi les deux acceptions suivantes de la nature conventionnelle 
du signe: a) «referring to an object by virtue of a convention» et b) «interpretable on the grounds of a convention».

En s'inspirant de ces définitions de la conventionnalité du signe, on peut affirmer en pratique qu'une traduction est conventionnelle au premier sens défini cidessus lorsqu'elle réfère à une unité ou un segment de la LD en vertu d'une convention. De même, une traduction est dite conventionnelle au deuxième sens défini ci-dessus lorsqu'elle procède de l'identité ou de la similitude du sens conventionnel de l'unité ou du segment en LA avec l'unité ou le segment en LD. Ces deux «méthodes» traduisent bien l'essentiel des mécanismes de transfert analogique en traduction: par la relation entre deux signes et par la relation entre deux sens.

Ces mécanismes de transfert distincts mais complémentaires de la conventionnalité de la traduction sont illustrés de façon exemplaire par les aléas de la traduction des sigles anglais naturalisés en français comme UNESCO, RAM, IP, IFAC qui possèdent pourtant une appellation propre en français: «Organisation des nations Unies pour l'éducation, la science et la culture», «mémoire vive», "protocole Internet», «Fédération internationale des experts-comptables». Comme la traduction de ces sigles procède d'un report (au sens de Delisle 1993) la traduction est conventionnelle au premier sens défini ci-dessus, c'est-à-dire par référence ou par correspondance qui ne donne lieu à aucun calcul sémiotique. Inversement, on trouve des traductions de sigles ou d'acronymes anglais qui découlent d'un procédé conventionnel de traduction qui repose sur le procédé conventionnel de formation des sigles en français, comme ONU, MEV, FSI, ICCA qui désignent respectivement "Organisation des Nations unies ", «mémoire vive», «fournisseur de service Internet» et «Institut canadien des comptables agréés». Devant cette alternative et face à un sigle moins fréquent, les traducteurs font face à un véritable arbitraire traductologique au sens défini ci-dessus puisque ces deux modes de traduction sont aujourd'hui en concurrence. Les traducteurs n'ont d'autre choix que de s'en remettre à l'usage, et à l'usage du client en particulier.

On peut ainsi définir deux modes conventionnels de traduction: un premier mode de correspondance qui s'appuie sur les traductions déjà établies et reconnues comme telles ${ }^{2}$ et un deuxième mode qui procède de l'équivalence et qui s'appuie sur un «calcul» sémiotique et sémantique des unités de la LA dans la reproduction de la signification du segment en LD.

\section{Les particularités de la traduction des UP}

La traduction des UP est d'abord particulière dans la mesure où elle comporte souvent la traduction d'une métaphore. Celle-ci ne fait cependant pas partie des caractéristiques inhérentes aux UP puisque ces dernières sont définies par la lexicalisation de leur sens, ce qui n'est pas nécessairement vrai des métaphores. Delisle (1993) définit au moins deux types de métaphores parmi celles qui intéressent les UP: les métaphores mortes et les métaphores figées ${ }^{3}$. Dans les deux cas, la nature métaphorique du sens des UP n'est pas incompatible avec la lexicalisation du sens des UP.

Les métaphores mortes sont les métaphores dont l'effet métaphorique banalisé dans le sens d'une unité lexicale fait partie de l'une de ses acceptions à titre de sens propre. L'exemple par excellence est celui de la catachrèse "pied (de chaise)» qui désigne, par métaphore anatomique banalisée, l'un des quatre supports d'une chaise. 
Ce type de métaphores se traduit comme une unité lexicale, et non pas comme un prédicat métaphorique. Les métaphores figées peuvent être considérées comme des UP puisque celles-ci ont un sens lexicalisé, par définition. À l'exception des difficultés de transfert de la métaphore, qui sont décrites ci-après, la principale difficulté qu'elles soulèvent est leur délimitation au sein même de l'UP qu'il importe de distinguer de la délimitation des UP elles-mêmes.

Soit l'exemple de Delisle (1993: 132) visant à montrer la différence entre l'équivalence et la correspondance: "help pay the rent», "aida à payer le loyer» et «réussit à joindre les deux bouts ». Ces trois expressions possèdent la même structure syntaxique, à une préposition près, et l'analyse de l'effet métaphorique aboutit à la même délimitation de la métaphore, à savoir après le premier verbe et son régime (la préposition) qui sert à exprimer une modalité du prédicat métaphorique qui suit. L'analyse de la traduction de ces expressions doit permettre de dégager deux éléments essentiels qui d'ailleurs se traduisent en deux étapes distinctes: la traduction de la métaphore, et de l'UP', dans ce cas-ci le prédicat métaphorique "pay the rent», et ensuite la traduction de l'expression de la modalité («help»). Même si dans ces expressions ces deux éléments se succèdent dans le temps, leur traduction doit suivre l'ordre inverse, c'est-à-dire le prédicat métaphorique avant l'expression de la modalité. Les deux traductions en français semblent avoir été réalisées suivant le même procédé de traduction, la différence se situant plutôt dans la nature non conventionnelle et conventionnelle de son résultat.

\section{Le caractère arbitraire de la traduction des UP}

L'arbitraire de la traduction des UP se vérifie dans le cas des nombreuses variantes des UP. En dépit de leur caractère conventionnel, il est surprenant de constater combien des éléments aussi conservateurs que les UP peuvent se manifester par des moyens linguistiques aussi variés. C'est le cas, par exemple, des expressions que l'on retrouve en français pour traduire "go at a snail's pace»: "avancer / rouler comme un escargot / une limace», «avancer / marcher à pas de tortue» ou " avancer à petits pas ». Dans ce dernier cas, à moins que le contexte ne neutralise la différence de sens et que celle-ci ne soit plus pertinente, on ne peut dire que la traduction par «avancer à petits pas» soit arbitraire puisqu'elle introduit une différence de sens perceptible.

Bien que ces exemples témoignent d'un arbitraire établi, ils n'expliquent cependant pas l'originalité de l'arbitraire du transfert des UP. On doit à Nida et Taber (1969: 106) la reconnaissance implicite du caractère arbitraire des UP dans la reconnaissance explicite de trois types possibles de «semantic adjustments» dans la traduction des UP, ou, en anglais usuel, des idioms: "The adjustments are quite understandably of three types: (a) from idioms to nonidioms, (b) from idioms to idioms, and (c) from nonidioms to idioms. " Le transfert d'une UP à une expression non phraséologique, et inversement d'une expression non phraséologique à une UP illustre donc que les périphrases sémantiques définitionnelles peuvent toujours servir à traduire une UP et que le caractère phraséologique n'est jamais obligatoirement transféré de la LD à la LA. 


\section{La nature conventionnelle de la traduction des UP}

Définie comme un calcul sémiotique du sens métaphorique de l'expression, l'équivalence est le procédé de traduction par excellence des UP. Le rôle important que joue le calcul sémiotique dans la traduction des UP découle de la nature arbitraire de la traduction des UP qui fait en sorte qu'une UP peut toujours être traduite par une périphrase sémantique, cette dernière étant même obligatoire dans l'interprétation de l'UP lorsque le sens de celle-ci est opaque. La traduction des UP est donc conventionnelle dans le deuxième sens de l'adjectif indiqué ci-dessus parce que la traduction d'une UP procède d'une identité de sens entre les segments en LA avec le sens du segment en LD. Cependant, dans la mesure où la correspondance ne se limite pas à la littéralité, ce procédé de traduction peut très bien servir aussi à la traduction des UP. Autrement dit, selon les connaissances et l'expérience de la personne qui traduit, la correspondance peut aussi servir à la traduction d'une UP qu'elle a déjà traduite. Étant donné que les UP sont des unités lexicales, et puisque la correspondance et l'équivalence peuvent être des procédés de traduction des UP, celle-ci est donc conventionnelle dans les deux sens de l'adjectif définis ci-dessus.

Les UP se traduisent de deux façons différentes selon que la métaphore qu'elles renferment s'étend à l'ensemble de l'UP ou qu'elle ne représente qu'une partie de celleci. Dans les deux cas l'UP est souvent associée à un élément linguistique obligatoire mais variable servant d'ancrage de l'UP dans le discours. Ce peut être le sujet, comme c'est le cas pour toutes les UP prédicatives, un complément du verbe ou un complément d'un nom. Ces éléments font nécessairement partie de l'UP même s'ils sont variables. Pour cette raison, ces derniers ne sont pas lexicalisés mais «grammaticalisés». Dans la discussion qui suit, la traduction de ces éléments n'est pas étudiée puisqu'ils se traduisent comme n'importe quelle autre unité lexicale ou expression linguistique non phraséologique.

Les UP dont la métaphore est globale se traduisent de façon conventionnelle suivant les procédés habituels de traduction, à savoir la correspondance, qui s'appuie sur les traductions déjà établies, ou l'équivalence, qui repose sur un calcul sémiotique du sens des expressions. À titre d'exemple, les expressions «kick the bucket» $=$ «casser sa pipe» et «hold one's horses» = "y aller doucement», pour lesquelles la correspondance comme l'équivalence sont possibles. Comme la première traduction est opaque, du fait notamment qu'elle est hors contexte et qu'elle n'est pas littérale, la correspondance est plus vraisemblable. Dans le deuxième exemple par contre, l'équivalence est plus probable du fait que la traduction en français est une périphrase sémantique pas du tout métaphorique (l'effet de sens est ici pragmatique).

Les UP dont la métaphore lexicalisée n'est pas globale se traduisent elles aussi de façon conventionnelle. Ce type d'UP représente le type le plus courant d'UP et de ce fait, le plus caractéristique. Même s'il n'est pas métaphorique, l'élément qui se trouve en dehors de la métaphore entretient toutefois des liens privilégiés avec la partie métaphorique de l'UP et peut être considéré pour cette raison comme une collocation de celle-ci. Contrairement cependant à l'ancrage, qui est grammaticalisé, cet élément non métaphorique est lexicalement figé. On peut donc considérer cet élément non métaphorique comme une collocation de la partie métaphorique de l'UP, d'autant plus que, contrairement à l'ancrage, celui-ci est toujours lexicalement restreint ${ }^{5}$. 
La traduction de l'élément non métaphorique joue un rôle aussi déterminant que celle de la métaphore. En effet, c'est sur cet élément que s'appuie le plus souvent la nature conventionnelle de la traduction. À titre d'exemple, bien que les segments «to the wolves» et «en pâture» ne soient pas des traductions conventionnelles de l'un et de l'autre, c'est la présence des prédicats «throw $s b$ » et «jeter qqn» qui sert de support à la métaphore et donc à la traduction conventionnelle. Il n'y a donc pas correspondance conventionnelle entre «to the wolves» et "en pâture», mais équivalence conventionnelle de celles-ci dans le cotexte «throw sb» et «jeter qqn». Ce qui n'empêche pas en outre l'établissement d'une correspondance conventionnelle portant sur la totalité des segments obligatoires dans les deux expressions.

La traduction de l'ensemble de l'UP «throw sb to the wolves» = «jeter qqn en pâture» est donc une traduction complexe qui implique la traduction d'au moins trois segments différents: la traduction de l'ancrage (le sujet du prédicat) qui est grammaticalisé (et obligatoire), et la traduction d'un élément figé complexe comprenant lui-même une métaphore et un collocatif servant de support à la métaphore, ce dernier élément se traduisant par correspondance conventionnelle. Autrement dit, l'élément métaphorique se traduit par équivalence «fonctionnelle» dans un cotexte linguistique extrêmement contraint qui joue tout à fait le rôle de la base dans une relation de collocation, par opposition au rôle du collocatif.

\section{Conséquences didactiques}

Comme l'explique Jean Delisle (1993: 387), un des premiers apprentissages de la traduction des UP est celle de leur reconnaissance en LD. Celle-ci nécessite une analyse du sens global et analytique des UP qui implique non seulement la délimitation de la métaphore dans l'UP, mais aussi la délimitation de l'UP dans la phrase. En outre, il faut aussi départager au sein même de l'UP, les éléments qui appartiennent à l'ancrage (les éléments grammaticalisés) des éléments proprement lexicalisés, et au sein de ces derniers, les éléments collocatifs des éléments métaphoriques. La compréhension des UP est donc une tâche complexe parce qu'elle implique une analyse fine des éléments grammaticalisés, lexicalisés et métaphoriques d'une expression linguistique. Dans cette tâche, le recours à l'analyse du sens et de la combinatoire syntaxique sont des outils absolument indispensables pour dissocier toutes les composantes des UP. Du point de vue pédagogique et didactique, le premier enseignement que l'on doit privilégier dans la traduction des UP est donc celui de la reconnaissance des UP et de leur analyse syntaxique et sémantique. Pour ce faire, on devra privilégier la consultation de dictionnaires spécialisés d'UP anglaises ou la réalisation d'exercices pratiques de traduction d'UP en classe ou en petits groupes. Dans ce dernier type d'exercice, on pourrait distinguer les exercices d'interprétation des UP à réaliser avec des étudiants qui débutent en traduction des exercices de traduction proprement dit que l'on peut réserver à des étudiants d'un niveau plus avancé.

Enfin, une deuxième difficulté de la traduction des UP consiste à ne pas oublier que si en LD l'on peut se contenter d'une simple reconnaissance des différents éléments que l'on retrouve dans une UP, il en va tout autrement en LA où les traducteurs doivent pouvoir compter sur une connaissance active des usages conventionnels phraséologiques et métaphoriques. À ce sujet, la traduction des UP offre un autre parallèle avec l'apprentissage des langues où l'on sait depuis longtemps que la 
connaissance active d'une langue diffère grandement d'une connaissance passive de celle-ci. Il importe donc de sensibiliser les étudiants aux emplois abusifs qui témoignent d'une mauvaise connaissance du bagage francophone et de sa composante phraséologique (proverbes, locutions, collocations, etc.).

\section{Conséquences théoriques}

Le recours aux deux sens de l'adjectif conventionnel en sémiotique a permis de redéfinir les procédés de traduction que sont la correspondance et l'équivalence. Tandis que la correspondance est établie à partir d'une relation de référence entre un segment ou une unité lexicale de la LD et un segment ou une unité lexicale de la LA, l'équivalence procède plutôt d'un calcul du sens du segment ou de l'unité lexicale de la LD et de sa réexpression en LA. À l'opposé de la correspondance qui revêt un caractère simplement mnémonique, l'équivalence repose sur une analyse ou une comparaison des sens en LD et en LA. Le procédé de transfert proprement dit semble donc être réservé à l'équivalence puisque la correspondance s'appuie sur une traduction, et peut-être, une équivalence, déjà établie. La distinction entre les notions de correspondance et d'équivalence pourrait donc s'expliquer par le caractère original du transfert sémantique, par opposition à un simple report qui ne procède pas d'une analyse du sens. Ainsi, on peut montrer que, même si les notions de correspondance et d'équivalence sont oppositives, il en va tout autrement de leur application sur un segment à traduire qui peut varier en fonction des besoins et des connaissances des traducteurs et des contingences contextuelles de la traduction (outils mis à la disposition de la traduction et, éventuellement, mémoire particulière de traduction constituée de textes choisis).

\section{Conclusion}

Nous avons montré tout d'abord que la traduction des UP est foncièrement arbitraire parce qu'elle peut toujours être sémantique et ne pas reproduire le caractère phraséologique ou métaphorique de la LD. En outre, la traduction des UP est doublement conventionnelle d'un point de vue sémiotique: d'une part du point de vue de la relation référentielle conventionnelle entre deux unités lexicales de langues différentes, et d'autre part du point de vue des relations sémantiques entre deux expressions de langues différentes, c'est-à-dire par la nature conventionnelle de l'équivalence qui s'appuie sur une relation d'identité de sens s'exprimant de façon conventionnelle.

La particularité de la traduction des UP tient au fait que le procédé d'équivalence des UP peut aussi être considéré comme une correspondance dans la mesure où la traduction est stable et générale, et dans la mesure aussi où la correspondance ne se limite pas à la traduction littérale. Cette particularité de la traduction des UP s'explique certainement par le fait que celles-ci sont des unités lexicales, sauf peutêtre en ce qui a trait à leur caractère arbitraire. Du fait que la traduction des UP est arbitraire et conventionnelle, les méthodes d'enseignement d'une langue étrangère ont beaucoup à offrir aux étudiants dans l'apprentissage de la traduction des UP et la théorie de la traduction a beaucoup à gagner, du point de vue de l'adéquation entre la théorie et la pratique, à envisager les notions d'équivalence et de correspondance non pas comme des oppositions, mais comme des méthodes ou procédés complémentaires de traduction. 


\section{NOTES}

1. Les autres procédés de traduction, comme l'économie et l'étoffement, sont plutôt le fruit d'une comparaison a posteriori de la traduction et ne décrivent pas le procédé lui-même. On ne saurait traduire par économie ou par étoffement (mais de manière étoffée et économe, si). On traduit par correspondances et équivalences.

2. Qui couvre davantage de traductions que le simple report d'une forme en LD en LA défini dans Jean Delisle (1993).

3. L'auteur distingue aussi les métaphores vives qui désignent une métaphore originale. Comme le sens de ces métaphores n'est par définition pas lexicalisé, celles-ci ne peuvent être considérées comme des UP.

4. Dans cet exemple, la délimitation de la métaphore et de l'UP coïncident.

5. La considération des difficultés de traduction et de définition des collocations n'a pas sa place ici, mais il est intéressant de signaler que les notions de collocation et d'UP sont intimement liées, à l'intérieur d'une même chaîne segmentale, contrairement à la présentation traditionnelle de ces éléments qui insiste plutôt sur leur caractère complémentaire, mais certainement pas combinatoire.

\section{RÉFÉRENCES}

Benveniste, E. (1966) : Problèmes de linguistique générale, volume I, Paris, Gallimard, coll. «Tel», $356 \mathrm{p}$.

Delisle, J. (1993): La traduction raisonnée. Manuel d'initiation à la traduction professionnelle de l'anglais vers le français, Ottawa, Les Presses de l'Université d'Ottawa, coll. «Pédagogie de la traduction », $486 \mathrm{p}$.

NidA, E. A. et C. R. TABer (1969): The Theory and Practice of Translation, Leyde, E. J. Brill, vin$220 \mathrm{p}$.

Poirier, E. (1995): Le Bestiaire: Dictionnaire bilingue et analogique des expressions animalières de la langue anglaise, Brossard (Québec), Linguatech, xxxıII-230 p.

Seвеок, T. A. (dir.) (1986) : Encyclopedic Dictionary of Semiotics, New York, Mouton de Gruyter, coll. «Approaches to Semiotics», $\mathrm{n}^{\circ}$ 73, 3 vol.

Vinay, J.-P. et J. Darbelnet (1958): Stylistique comparée du français et de l'anglais: méthode de traduction, nouv. éd. rev. et corr., Montréal, Beauchemin, 331 p. 